\title{
Set Contractions and KKM Mappings in Banach Spaces
}

\author{
A. Razani and N. Karamikabir \\ Department of Mathematics, Karaj Branch, Islamic Azad University, Karaj, Iran \\ Correspondence should be addressed to A. Razani; razani@ipm.ir
}

Received 10 April 2013; Accepted 17 June 2013

Academic Editor: Chengming Huang

Copyright (C) 2013 A. Razani and N. Karamikabir. This is an open access article distributed under the Creative Commons Attribution License, which permits unrestricted use, distribution, and reproduction in any medium, provided the original work is properly cited.

Some fixed point theorems for generalized set contraction maps and KKM type ones in Banach spaces are presented. Moreover, a new generalized set contraction is introduced. As an application, some coincidence theorems for KKM type set contractions are obtained.

\section{Introduction}

Let $E$ be a Banach space and

$$
\begin{aligned}
& \mathscr{P}_{p}(E) \\
& \quad=\{A \subset E: A \text { is a nonempty and has a property } p\} .
\end{aligned}
$$

Thus $\mathscr{P}_{\mathrm{bd}}(E), \mathscr{P}_{\mathrm{cl}}(E), \mathscr{P}_{\mathrm{cv}}(E), \mathscr{P}_{\mathrm{cp}}(E), \mathscr{P}_{\mathrm{co}}(E), \mathscr{P}_{\mathrm{cl}, \mathrm{bd}}(E)$, $\mathscr{P}_{\text {cp,cv }}(E)$, and $\mathscr{P}_{\text {rcp }}(E)$ denote the classes of all bounded, closed, convex, compact, connected, closed-bounded, compact-convex, and relatively compact subsets of $E$, respectively [1]. Let $E_{1}$ and $E_{2}$ be two Banach spaces. A multivalued mapping $T: E_{1} \rightarrow \mathscr{P}_{p}\left(E_{2}\right)$ is said to be

(i) upper semicontinuous if and only if for every closed subset $B$ of $E_{2}$, the set $T^{-}(B)=\left\{x \in E_{1}: T(x) \cap B \neq \emptyset\right\}$ is a closed subset of $E_{1}$,

(ii) closed if its graph $\operatorname{Gr}(T)=\left\{(x, y) \in E_{1} \times E_{2}: y \in\right.$ $T(x)\}$ is a closed subset of $E_{1} \times E_{2}$,

(iii) compact if $\overline{T\left(E_{1}\right)}$ is a compact subset of $E_{2}$.

The first fixed point theorem for multivalued mappings is due to Kakutani in Banach spaces, in 1941 [2]. He proved a generalization of Brouwer's fixed point theorem to the multivalued mappings.

Theorem 1 (see [2]). Let $K$ be a compact subset of a Banach space $E$ and let $F: K \rightarrow \mathscr{P}_{c v, c p}(K)$ be an upper semicontinuous multivalued operator. Then $F$ has a fixed point.
The above theorem has been extended in the literature by generalizing or modifying the domain space $E$, domain set $K$, and the nature of the multivalued operator $F$. Here, compactness plays an essential role. The following definition of measure of noncompactness on a bounded subset of the Banach space $E$ is given by Dhage in 2010 .

Definition 2 (see [1]). A function $\mu: \mathscr{P}_{\text {bd }}(E) \rightarrow \mathbb{R}^{+}$is called a measure of noncompactness if it satisfies

(i) $\emptyset \neq \mu^{-1}(0) \subset \mathscr{P}_{\text {rcp }}(E)$,

(ii) if $A \subseteq B$ then $\mu(A) \leq \mu(B)$,

(iii) $\mu(\bar{A})=\mu(A)$, where $\bar{A}$ denotes the closure of $A$,

(iv) $\mu(\operatorname{Conv}(A))=\mu(A)$, where $\operatorname{Conv}(A)$ denotes the convex hull of $A$,

(v) if $\left\{A_{n}\right\}$ is a decreasing sequence of sets in $\mathscr{P}_{\text {cl,bd }}(E)$ satisfying $\lim _{n \rightarrow \infty} \mu\left(A_{n}\right)=0$, then the limiting set $A_{\infty}=\cap_{n=1}^{\infty} \overline{A_{n}}$ is nonempty.

Definition 3 (see [1]). A multivalued mapping $T: E \rightarrow$ $\mathscr{P}_{\text {cl,bd }}(E)$ is called $\mathscr{D}$-set Lipschitz if there exists a continuous nondecreasing function $\psi: \mathbb{R}^{+} \rightarrow \mathbb{R}^{+}$such that $\mu(T(A)) \leq$ $\psi(\mu(A))$ for all $A \in \mathscr{P}_{\text {cl,bd }}(E)$ with $T(A) \in \mathscr{P}_{\text {cl,bd }}(E)$, where $\psi(0)=0$. Sometimes we call the function $\psi$ to be a $\mathscr{D}$-function of $T$ on $E$. In the spatial case, when $\psi(r)=k r, k>0, T$ is called a $k$-set Lipschitz mapping and if $k<1$, then $T$ is called a $k$-set contraction on $E$. Further, if $\psi(r)<r$ for $r>0$, then $T$ is called a nonlinear $\mathscr{D}$-set contraction on $E$. 
Dhage proved a generalization of Theorem 1 under weaker upper semicontinuity conditions in 2010 [1].

Theorem 4. Let $X$ be a nonempty, closed, convex, and bounded subset of a Banach space E and let $T: X \rightarrow \mathscr{P}_{\mathrm{cl}, c v}(X)$ be a closed and nonlinear $\mathscr{D}$-set contraction. Then $T$ has a fixed point.

Lemma 5 (see [3]). If $\psi$ is a D-function with $\psi(r)<r$ for $r>0$, then $\lim _{n \rightarrow \infty} \psi^{n}(t)=0$ for all $t \in[0, \infty)$.

Recall that a function $\varphi: \mathbb{R}^{+} \rightarrow \mathbb{R}^{+}$is called a comparison function if $\varphi$ is increasing and $\lim _{n \rightarrow \infty} \varphi^{n}(t)=0$ for all $t \in \mathbb{R}^{+}[4]$. As a consequence, $\varphi(t)<t$ for any $t>0, \varphi$ is continuous at 0 , and $\varphi(0)=0$.

A function $\varphi: \mathbb{R}^{+} \rightarrow \mathbb{R}^{+}$is called a (c)-comparison function if $\varphi$ is increasing and there exist $k_{0} \in \mathbb{N}, a \in(0,1)$ and a convergent series of non-negative terms $\sum_{k=1}^{\infty} v_{k}$ such that

$$
\varphi^{k+1}(t) \leq a \varphi^{k}(t)+v_{k}
$$

for $k \geq k_{0}$ and any $t \in[0, \infty)[5]$. If $\varphi: \mathbb{R}^{+} \rightarrow \mathbb{R}^{+}$is a (c)comparison function, then $\varphi$ is a comparison function [5]. So, we can define $\mathscr{C}$-set contraction as follows.

Definition 6. A multivalued mapping $T: E \rightarrow \mathscr{P}_{\text {cl,bd }}(E)$ is called $\mathscr{C}$-set contraction if there exists a continuous (c)comparison function $\varphi$ such that $\mu(T(A)) \leq \varphi(\mu(A))$ for all $A \in \mathscr{P}_{\mathrm{cl}, \mathrm{bd}}(E)$ with $T(A) \in \mathscr{P}_{\mathrm{cl}, \mathrm{bd}}(E)$.

Let $(X, d)$ be a metric space. The Hausdorff metric $H_{d}$ induced by the metric $d$ and defined as follows

$$
H_{d}(A, B)=\max \left\{\sup _{a \in A} d(a, B), \sup _{b \in B} d(b, A)\right\} .
$$

Definition 7 (see [6]). Let $\varepsilon>0$. A multivalued contractive ( $\varepsilon$-contractive) map is a map $F: X \multimap \mathscr{P}_{\text {cl,bd }}(X)$ such that for all $x, y \in X$ with $x \neq y$ (and for some $\varepsilon>0, d(x, y)<\varepsilon$, resp.), $H_{d}(F(x), F(y))<d(x, y)$.

Theorem 8 (see [7]). Let $X$ be a nonempty compact and connected metric space and let $F: X \rightarrow \mathscr{P}_{c p}(X)$ be a multivalued $\varepsilon$-contractive map, then $F$ has a fixed point.

We need the following definitions of KKM theory in the sequel [8].

Assume that $X$ is a convex subset of a topological vector space and $Y$ is a topological space. If $G: X \rightarrow \mathscr{P}_{p}(Y)$ and $F: X \rightarrow \mathscr{P}_{p}(Y)$ are two multivalued maps and for each $A \in\langle X\rangle, F(\operatorname{Conv}(A)) \subset G(A)$, then $G$ is called generalized KKM mapping with respect to $F$, where $\langle X\rangle$ denote the family of all nonempty finite subsets of $X$. More generally, if $F: X \rightarrow \mathscr{P}_{p}(Y)$ satisfies the requirement that for any generalized KKM mapping $G: X \rightarrow \mathscr{P}_{p}(Y)$ with respect to
$F$ and the family $\{\overline{G(x)}: x \in X\}$ has the finite intersection property, then $F$ is said to have the KKM property. Let

$$
\begin{aligned}
& \operatorname{KKM}(X, Y) \\
& \quad=\left\{F: X \rightarrow \mathscr{P}_{p}(Y): F \text { has the KKM property }\right\} .
\end{aligned}
$$

Lemma 9 (see [8]). Let $X$ be a nonempty convex subset of Hausdorff topological vector space $E$. Then $\left.T\right|_{D} \in \operatorname{KKM}(D, Y)$ whenever $T \in K K M(X, Y)$ and $D$ is a nonempty convex subset of $X$.

Chen and Chang obtained some fixed point theorems for KKM type set contraction mappings in various spaces [912]. In 2010, Amini-Harandi et al. introduced generalized set contraction on topological spaces [13].

In Section 2, we present some fixed point theorems for generalized set contractions which are $\varepsilon$-contractive $(\mathrm{KKM} \varepsilon$ contractive) multivalued maps. In the first step of Section 3, we introduce a new type of generalized set contraction and then prove that the results of Section 2 hold for them. Section 4 is devoted to some KKM coincidence theorems as applications of these results.

\section{Generalized Set Contractions}

In this section by applying Theorem 8 , we obtain some fixed point theorems for $\varepsilon$-contractive multivalued maps which are either generalized set contraction or KKM type ones. In all cases, the multivalued maps are not necessarily compact values. We consider measurement of noncompactness in Definition 2.

Definition 10 (see [13]). A multivalued mapping $F: E \rightarrow$ $\mathscr{P}_{p}(E)$ is said to be a generalized set contraction, if for each $\epsilon>0$ there exists $\delta>0$ such that for $A \in \mathscr{P}_{\text {cl,bd }}(E)$ with $\epsilon \leq \mu(A)<\epsilon+\delta$, there exists $n \in N$ such that $\mu\left(F^{n}(A)\right)<\epsilon$.

Lemma 11 (see [13]). Let $X$ be a topological space and let $\mu$ be a measure of noncompactness on $X$. Suppose that $F$ is a generalized set contraction on $X$. Then for every subset $A$ of $X$ for which $F(A) \subset A$ and $\mu(A)<\infty$, one has $\lim _{n \rightarrow \infty} \mu\left(F^{n}(A)\right)=0$.

Proposition 12. Let $E$ be a Hausdorff topological space. If $\left\{X_{n}\right\}$ is a decreasing sequence of closed and connected sets in $\mathscr{P}_{c l, b d}(E)$ such that $\lim _{n \rightarrow \infty} \mu\left(X_{n}\right)=0$, then $\cap_{n=1}^{\infty} X_{n}$ is nonempty, compact, and connected.

Proof. Clearly, $K=\cap_{n=1}^{\infty} X_{n}$ is a nonempty, closed, and compact subset of $E$. Let $A$ and $B$ be two nonempty, disjoint, and closed sets so that $K=A \cup B$. We can find disjoint open sets $U$ and $V$ around $A$ and $B$, respectively. For every $n \in \mathbb{N}, X_{n} \backslash(U \cup V)$ is nonempty. If not, then $\left(U \cap X_{n}\right)$ and $\left(V \cap X_{n}\right)$ are nonempty and $X_{n}=\left(U \cap X_{n}\right) \cup\left(V \cap X_{n}\right)$, which cannot happen. The collection of $\left\{X_{n} \backslash(U \cup V)\right\}$ is also a decreasing sequence of nonempty closed sets. Since $\mu\left(X_{n} \backslash(U \cup V)\right) \leq \mu\left(X_{n}\right)$ then $\mu\left(X_{n} \backslash(U \cup V)\right) \rightarrow 0$ as $n \rightarrow \infty$. Hence, $\cap_{n=0}^{\infty}\left(X_{n} \backslash(U \cup V)\right)$ is nonempty, that is, $K \cap(U \cup V)^{c} \neq \emptyset$, which is a contradiction. 
Theorem 13. Let $X$ be a nonempty, bounded, closed, and connected subset of Banach space E. If $F: X \rightarrow \mathscr{P}_{c l, c o}(X)$ is an $\varepsilon$-contractive and generalized set contraction, then $F$ has a fixed point and the set of fixed points of $F$ is compact.

Proof. Let $X_{0}=X$ and $X_{n}=\overline{F^{n}(X)}$ for all $n \in \mathbb{N}$. Since $F$ is an $\varepsilon$-contractive map, then $F$ is continuous and $F(\bar{A}) \subseteq \overline{F(A)}$. On the other hand, $F^{n+1}(X) \subseteq F^{n}(X)$, so we have $F\left(X_{n}\right) \subseteq X_{n}$ and $X_{n+1} \subseteq X_{n}$. Since $F$ is continuous with closed and connected values, then by [14, Lemma 1.6], $F(X)$ is connected. Hence, $X_{n}$ which is closure of connected set $F^{n}(X)$ for all $n \in \mathbb{N}$, is connected. But $\mu\left(X_{n}\right)=\mu\left(F^{n}(X)\right)$, and by Lemma 11 we have $\mu\left(X_{n}\right) \rightarrow 0$. Therefore, by Proposition $12, K=\cap_{n=1}^{\infty} X_{n}$ is nonempty compact and connected. Since $F(K) \subseteq K$, then the desired conclusion followed by an application of Theorem 8 to the multivalued map $F: K \rightarrow \mathscr{P}_{\text {cp }}(K)$.

Let $B=\{x \in X: x \in F(x)\}$. We claim that $\epsilon_{0}=\mu(B)=0$. If $\mu(B) \neq 0$ then $\mu(B)=\mu\left(F^{n}(B)\right)$. Since $F$ is a generalized set contraction, then for $\epsilon_{0}>0$, there exists $\delta>0$ such that for $B \subseteq X$ with $\epsilon_{0} \leq \mu(B)<\epsilon_{0}+\delta$, there exists $n \in \mathbb{N}$ such that $\epsilon_{0}=\mu(B)<\epsilon_{0}$, which is a contradiction. So $B$ is relatively compact, and since $F$ is continuous, then $B$ is a compact subset of $X$.

If $F: E \rightarrow \mathscr{P}_{p}(E)$ is a $k$-set contraction, then $F$ is a generalized set contraction, but the converse is not true [13]. Therefore, we have the following result.

Corollary 14. Let $X$ be a nonempty, bounded, closed, and connected subset of Banach space E. If $F: X \rightarrow \mathscr{P}_{c l, c o}(X)$ is an $\varepsilon$-contractive and $k$-set contraction, then $F$ has a fixed point.

Corollary 15. Let $X$ be a nonempty, closed, and bounded subset of Banach space E. If $F: X \rightarrow \mathscr{P}_{c l, b d}(X)$ is an $\varepsilon$ contractive and generalized set contraction, then there exists a compact subset $K$ of $X$ such that $F(K) \subseteq K$.

Theorem 16. Let $X$ be a nonempty, closed, and bounded subset of Banach space E. If $F \in K K M(X, X)$ is an $\varepsilon$-contractive and generalized set contraction with nonempty closed and bounded values, then $F$ has a fixed point.

Proof. By Corollary 15, there exists a compact subset $K$ of $X$ such that $F(K) \subseteq K$. Let $Y=\overline{F(K)}$. Hence there exists a finite subset $A$ of $K$ such that $Y \subseteq \cup_{x \in A} N(x, \varepsilon)$, where $N(x, \varepsilon)=\{y \in E:\|x-y\|<\varepsilon\}$. Define a map $T: K \rightarrow$ $\mathscr{P}_{p}(Y)$ by $T(x)=Y \backslash N(x, \epsilon)$ for all $x \in K$; then $T(x)$ is closed for each $x \in K$ and $\cap_{x \in A} T(x)=\emptyset$. Since $Y \subseteq$ $K \subseteq X$ and $F \in \operatorname{KKM}(X, X)$, then by Lemma $9,\left.F\right|_{K} \in$ $\operatorname{KKM}(K, Y)$. Thus, $T$ is not a generalized KKM map with respect to $\left.F\right|_{K}$. Hence, there exists $\left\{x_{0}, x_{1}, \ldots, x_{n}\right\}$ of $K$ such that $F\left(\operatorname{Conv}\left(\left\{x_{0}, x_{1}, \ldots, x_{n}\right\}\right)\right) \nsubseteq \cup_{i=0}^{n} T\left(x_{i}\right)$. Thus there exists $y \in F\left(\operatorname{Conv}\left(\left\{x_{0}, x_{1}, \ldots, x_{n}\right\}\right)\right)$ such that $y \notin \cup_{i=0}^{n} T\left(x_{i}\right)$, that is, $y \in F(z)$ for some $z \in \operatorname{Conv}\left(\left\{x_{0}, x_{1}, \ldots, x_{n}\right\}\right)$ and $y \in N\left(x_{i}, \epsilon\right)$, so $x_{i} \in N(y, \epsilon)$ for $i \in\{0,1, \ldots, n\}$. Since $z \in \operatorname{Conv}\left(\left\{x_{0}, x_{1}, \ldots, x_{n}\right\}\right) \subset B(y, \epsilon)$, then $y \in F(z) \cap B(z, \epsilon)$. Since $Y$ is a compact subset of $X$, then $y$ converges to some $x \in Y$ as $\epsilon \rightarrow 0$. Consequently, $z$ converges to $x$ as $\epsilon \rightarrow 0$.
Since $F$ is continuous, then by [14, Lemma 1.6] we have $x \in$ $F(x)$.

\section{Asymptotic Generalized Set Contractions}

In this section, we define a new type of set contraction in Banach spaces. Then we prove that the results of Section 2 hold for them. Also, we conclude some fixed point theorems for nonlinear $\mathscr{D}$-set contractions.

Definition 17. Let $X$ be a nonempty, closed, and bounded subset of a Banach space $E$. A multivalued mapping $F: X \rightarrow$ $\mathscr{P}_{p}(X)$ is said to be an asymptotic generalized set contraction, if there exists a sequence $\left\{\varphi_{n}\right\}$ of functions from $\mathbb{R}^{+}$in to itself satisfies

(i) for each $\varepsilon>0$, there exists $\delta>0$ and $m \in \mathbb{N}$ such that $\varphi_{m}(t) \leq \varepsilon$ for all $\varepsilon \leq t<\varepsilon+\delta$,

(ii) $\mu\left(F^{n}(X)\right)<\varphi_{n}(\mu(X))$.

Theorem 18. Let $X$ be a nonempty, bounded, closed, and connected subset of Banach space E. If $F: X \rightarrow \mathscr{P}_{c l, c o}(X)$ is an $\varepsilon$-contractive and asymptotic generalized set contraction, then $F$ has a fixed point.

Proof. Define a sequence $\left\{X_{n}\right\}$ of sets in $\mathscr{P}_{\text {cl,bd }}(X)$ such that $X_{0}=X$ and $X_{n}=\overline{F^{n}(X)}$ for all $n \in \mathbb{N}$. As the proof of Theorem 13, $F\left(X_{n}\right) \subseteq X_{n}, X_{n+1} \subset X_{n}$, and $X_{n}$ is connected for all $n \in \mathbb{N}$. If there exists an integer $N>0$ such that $\mu\left(X_{N}\right)=0$, then $X_{N}$ is a compact and connected set and invariant under $F$. Thus Theorem 8 implies that $F: X_{N} \rightarrow \mathscr{P}_{\text {cp }}\left(X_{N}\right)$ has a fixed point. So we assume that $\mu\left(X_{n}\right) \neq 0$ for all $n \in \mathbb{N}$. Define $\varepsilon_{n}=\mu\left(X_{n}\right)$ and $r=\inf \varepsilon_{n}$. If $r \neq 0$, by Definition 17, there exists $n_{0} \in \mathbb{N}, \delta_{r}>0$ and $m \in \mathbb{N}$ such that $\varphi_{m}(t) \leq r$ for all $r \leq t<r+\delta_{r}$ and $r \leq \varepsilon_{n_{0}}<r+\delta_{r}$, so

$$
\begin{aligned}
\varepsilon_{n_{0}+m} & =\mu\left(X_{n_{0}+m}\right)=\mu\left(F^{n_{0}+m}(X)\right) \\
& <\varphi_{m}\left(\mu\left(F^{n_{0}}(X)\right)\right)=\varphi_{m}\left(\mu\left(X_{n_{0}}\right)\right) \leq r,
\end{aligned}
$$

which is a contradiction. Hence $\mu\left(X_{n}\right) \rightarrow 0$ as $n \rightarrow \infty$. Now by Proposition 12, $K=\cap_{n=1}^{\infty} X_{n}$ is nonempty, compact, and connected. Moreover $F(K) \subseteq K$. So by Theorem 8 , the multivalued map $F: K \rightarrow \mathscr{P}_{\text {cp }}(K)$ has a fixed point.

Corollary 19. Let $X$ be a nonempty, closed, and bounded subset of Banach space E. If $F: X \rightarrow \mathscr{P}_{c l, b d}(X)$ is an $\mathcal{\varepsilon}$ contractive and asymptotic generalized set contraction, then there exists a compact subset $K$ of $X$ such that $F(K) \subseteq K$.

The proof of following theorem is similar to that of Theorem 16; hence it is omitted.

Theorem 20. Let $X$ be a nonempty, closed, and bounded subset of Banach space E. If $F \in K K M(X, X)$ is an $\varepsilon$-contractive and asymptotic generalized set contraction with nonempty closed and bounded values, then $F$ has a fixed point.

Proposition 21. Let $X$ be a nonempty, closed, and bounded subset of Banach space E. If $F: X \rightarrow \mathscr{P}_{c l, b d}(X)$ is a nonlinear 
D-set contraction, then $F$ is an asymptotic generalized set contraction.

Proof. Let $F$ be a nonlinear $\mathscr{D}$-set contraction with $\mathscr{D}$ function $\psi$. Define $\varphi_{n}=\psi^{n}$ for all $n \in \mathbb{N}$. Clearly, $\mu\left(F^{n}(X)\right) \leq$ $\psi^{n}(\mu(X))=\varphi_{n}(\mu(X))$, on the other hand, by Lemma 5 we have $\varphi_{n}(t) \rightarrow 0$ as $n \rightarrow \infty$. Thus $F$ is an asymptotic generalized set contraction.

Applying Proposition 21, Theorems 18 and 20, it is easy to conclude the following results.

Corollary 22. Let $X$ be a nonempty, bounded, closed, and connected subset of Banach space E. If $F: X \rightarrow \mathscr{P}_{c l, c o}(X)$ is an $\varepsilon$-contractive and nonlinear $\mathscr{D}$-set contraction, then $F$ has a fixed point.

Corollary 23. Let $X$ be a nonempty, closed, and bounded subset of Banach space E. If $F \in K K M(X, X)$ is an $\varepsilon$-contractive and nonlinear $\mathscr{D}$-set contraction with nonempty closed and bounded values, then $F$ has a fixed point.

Remark 24. Since every $\mathscr{C}$-set contraction is a nonlinear $\mathscr{D}$ set contraction, then Theorem 4, Corollaries 22 and 23 hold for these mappings.

\section{Some Applications in KKM Theory}

In this section we obtain two coincidence theorems for KKM type set contractions.

Theorem 25. Let $X$ be a nonempty, closed, bounded, and convex subset of Banach space E. If $F: X \rightarrow \mathscr{P}_{c l, b d}(X)$ and $G: X \rightarrow \mathscr{P}_{c v}(X)$ are two multivalued mappings satisfying

(i) $F \in K K M(X, X)$,

(ii) $F$ is a generalized (an asymptotic) set contraction and $\varepsilon$-contractive map,

(iii) for each compact subset $C$ of $X$ and any $y \in X, G^{-}(y) \cap$ $C$ is open in $C$,

then, there exists $x_{0}, y_{0} \in X$ such that $y_{0} \in F\left(x_{0}\right)$ and $x_{0} \in$ $G\left(y_{0}\right)$.

Proof. By Corollary 15 (Corollary 19), there exists a compact subset $K$ of $X$ such that $F(K) \subset K$. Since $G(x) \neq \emptyset$ and $K$ is compact, then $X=\cup_{x \in X} G^{-}(X)$ and $K=\cup_{i=1}^{n}\left(G^{-}\left(x_{i}\right) \cap K\right)$ for some $x_{1}, \ldots, x_{n} \in X$. Define a map $S: X \rightarrow \mathscr{P}_{p}(K)$ by $S(x)=K \backslash\left(G^{-}(x) \cap K\right)$ for each $x \in X$, then $\cap_{i=1}^{n} S\left(x_{i}\right)=\emptyset$. Therefore, $S$ is not a generalized KKM map with respect to $F$. So there exists a finite subset $A=\left\{a_{1}, \ldots a_{m}\right\}$ of $X$ such that $F(\operatorname{Conv}(A)) \nsubseteq S(A)$. Hence, there exist $x_{0} \in \operatorname{Conv}(A)$ and $y_{0} \in F\left(x_{0}\right)$ such that $y_{0} \notin S(A)$. Thus $y_{0} \in G^{-}\left(a_{i}\right) \cap K$ and so $a_{i} \in G\left(y_{0}\right)$ for $i=1, \ldots, m$. Since $G\left(y_{0}\right)$ is convex, then $\operatorname{Conv}(A) \subseteq G\left(y_{0}\right)$ and so $x_{0} \in G\left(y_{0}\right)$.

By Proposition 21, Corollary 19, and slight modification of the proof of Theorem 25, we have the following theorem.
Theorem 26. Let $X$ be a nonempty, closed, bounded, and convex subset of Banach space E. If $F: X \rightarrow \mathscr{P}_{c l, c v}(X)$ and $G: X \rightarrow \mathscr{P}_{c v}(X)$ are two multivalued mappings satisfying

(i) $F \in K K M(X, X)$ is a nonlinear $\mathscr{D}$-set contraction.

(ii) for each compact subset $C$ of $X$ and any $y \in X, G^{-}(y) \cap$ $C$ is open in $C$,

then, there exists $x_{0}, y_{0} \in X$ such that $y_{0} \in F\left(x_{0}\right)$ and $x_{0} \in$ $G\left(y_{0}\right)$.

\section{References}

[1] B. C. Dhage, "Some generalizations of mulit-valued version of Schauder's fixed point theorem with applications," Cubo, vol. 12, no. 3, pp. 139-151, 2010.

[2] S. A. Kakutani, "A generalization of Brouwer's fixed point theorem," Duke Mathematical Journal, vol. 8, pp. 457-459, 1941.

[3] B. C. Dhage, "Asymptotic stability of nonlinear functional integral equations via measures of noncompactness," Communications on Applied Nonlinear Analysis, vol. 15, no. 2, pp. 89-101, 2008.

[4] I. A. Rus, Generalized Contractions and Applications, Cluj University Press, Cluj-Napoca, Romania, 2001.

[5] V. Berinde, Contracţii generalizate şi apliciţtii, vol. 22, Editura Cub Press, Baia Mare, Romania, 1997.

[6] M. Edelstein, "On fixed and periodic points under contractive mappings," Journal of the London Mathematical Society, vol. 37, pp. 74-79, 1962.

[7] S. B. Nadler Jr., "Periodic points of multi-valued $\epsilon$-contractive maps," Topological Methods in Nonlinear Analysis, vol. 22, no. 2, pp. 399-409, 2003.

[8] T.-H. Chang and C.-L. Yen, "KKM property and fixed point theorems," Journal of Mathematical Analysis and Applications, vol. 203, no. 1, pp. 224-235, 1996.

[9] T. H. Chang and C. M. Chen, "Fixed point theorems for the generalized $\Psi$-set contraction mapping on an abstract convex space," Taiwanese Journal of Mathematics, vol. 14, no. 5, pp. 2015-2025, 2010.

[10] C.-M. Chen and T.-H. Chang, "Some results for the family $\operatorname{KKM}(X, Y)$ and the $\Phi$-mapping," Journal of Mathematical Analysis and Applications, vol. 329, no. 1, pp. 92-101, 2007.

[11] C.-M. Chen and T.-H. Chang, "Fixed point theorems for a weaker Meir-Keeler type $\psi$-set contraction in metric spaces," Fixed Point Theory and Applications, vol. 2009, Article ID 129124, pp. 1-8, 2009.

[12] C.-M. Chen, "Fixed point theorems for cyclic Meir-Keeler type mappings in complete metric spaces," Fixed Point Theory and Applications, vol. 2012, article 41, pp. 1-13, 2012.

[13] A. Amini-Harandi, M. Fakhar, and J. Zafarani, "Fixed point theorems for generalized set-contraction maps and their applications," Nonlinear Analysis: Theory, Methods \& Applications , vol. 72, no. 6, pp. 2891-2895, 2010.

[14] R. E. Smithson, "Some general properties of multi-valued functions," Pacific Journal of Mathematics, vol. 15, no. 2, pp. 681703, 1965. 


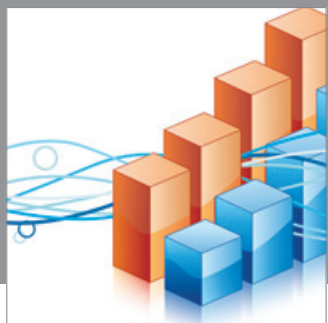

Advances in

Operations Research

mansans

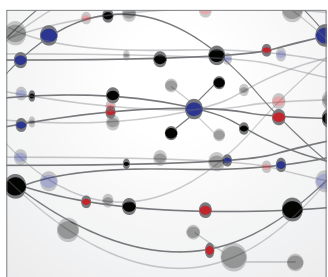

The Scientific World Journal
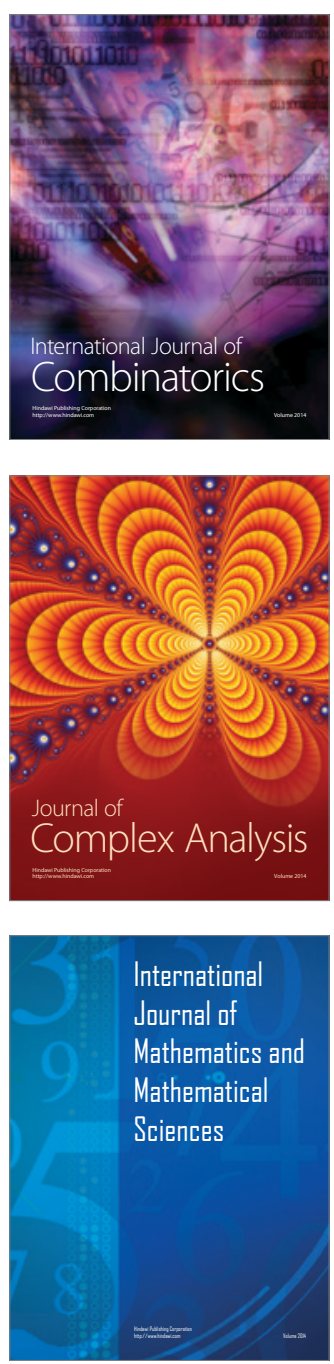
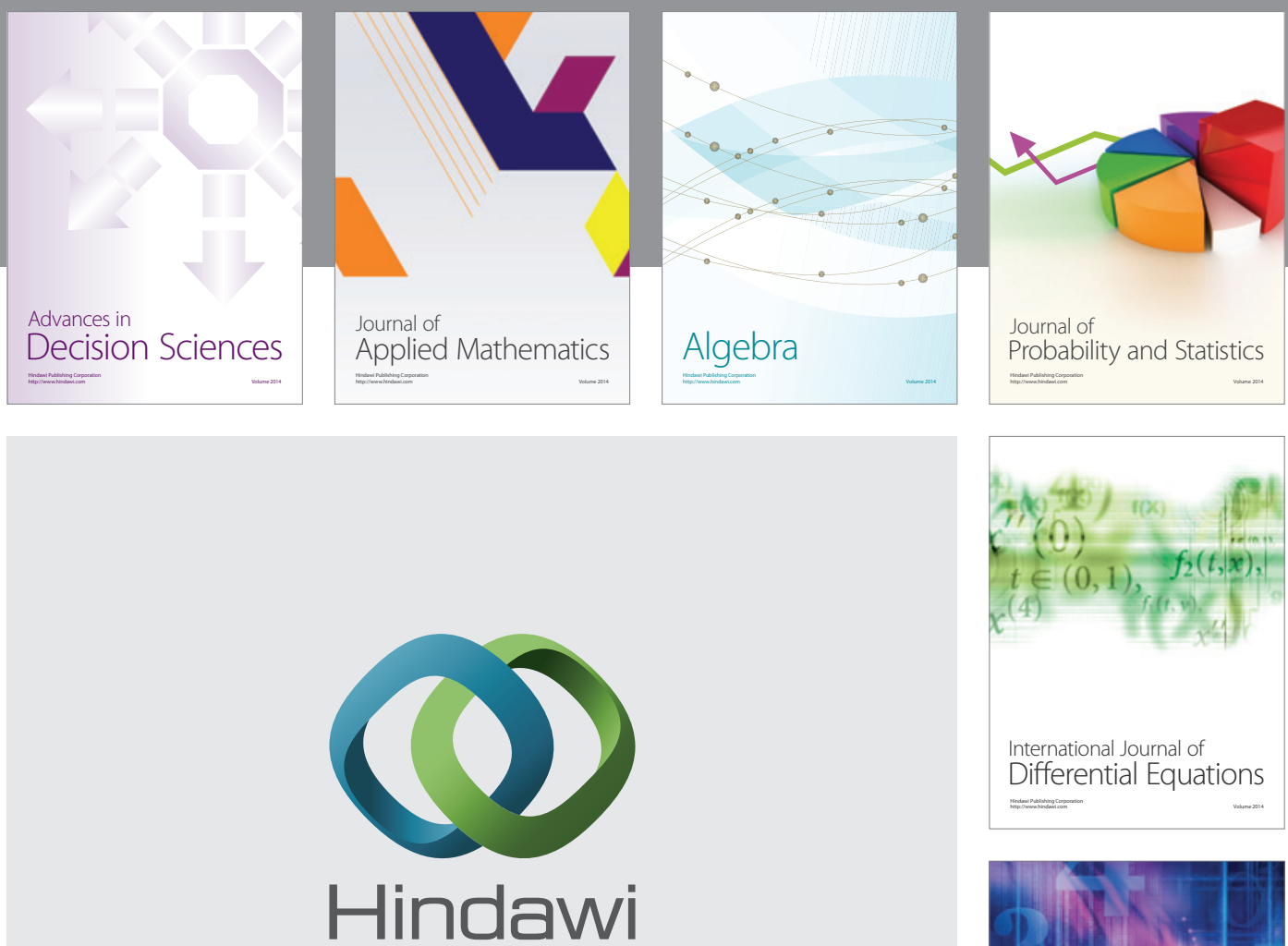

Submit your manuscripts at http://www.hindawi.com
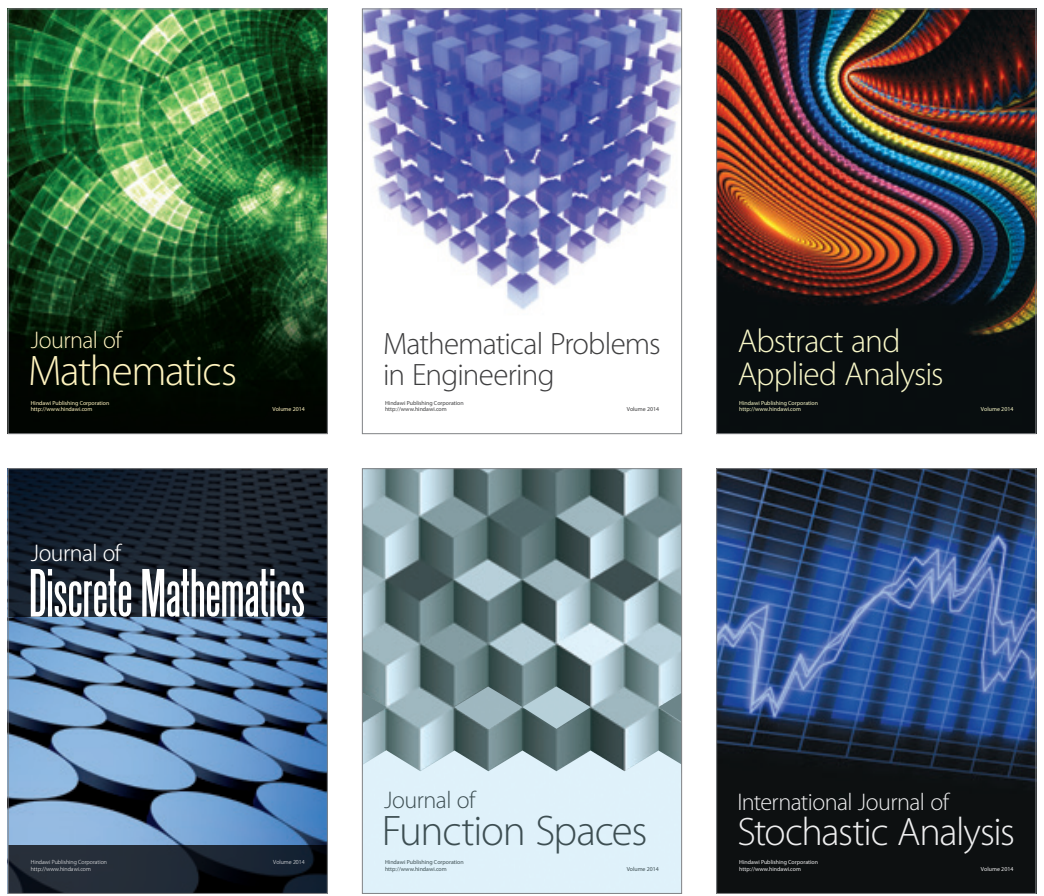

Journal of

Function Spaces

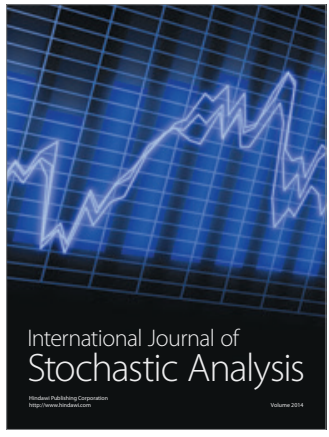

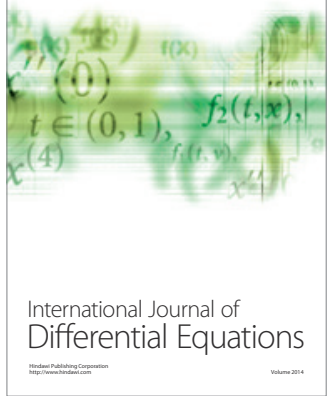
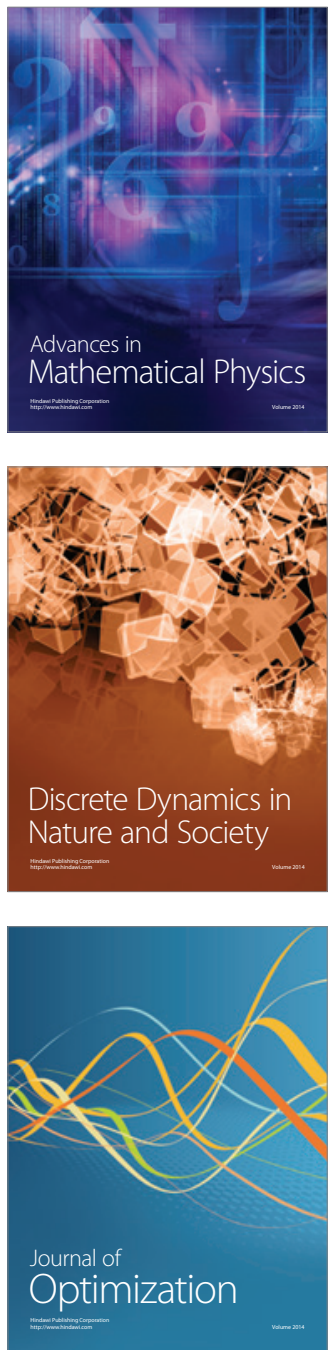